\title{
Distal 7q11.23 microduplication syndrome
}

INSERM

\section{Source}

INSERM. (1999). Orphanet: an online rare disease and orphan drug data base. Distal 7q11.23 microduplication syndrome. ORPHA:261102

Distal 7q11.23 microduplication syndrome is a rare chromosomal anomaly characterized by a predominantly neuropsychiatric phenotype with a few dysmorphic characteristics. Speech delay, learning difficulties, attention deficit hyperactivity disorder, bipolar disorder and aggressiveness have been reported. 